\title{
Young adults with spina bifida transitioned to a medical home: a survey of medical care in Jacksonville, Florida
}

\author{
Antonio M. Aguilera, ${ }^{1}$ David L. Wood, MD, MPH, ${ }^{2}$ Cortney Keeley, PA-C, ${ }^{3}$ Hector E. James, MD, ${ }^{4}$ \\ and Philipp R. Aldana, MD ${ }^{4}$ \\ ${ }^{1}$ University of Florida College of Medicine, Gainesville, Florida; ${ }^{2}$ Department of Pediatrics, Quillen College of Medicine, East \\ Tennessee State University, Johnson City, Tennessee; and 'Division of Community and Societal Pediatrics, Department of \\ Pediatrics, and ${ }^{4}$ Division of Pediatric Neurosurgery, Department of Neurosurgery, University of Florida College of Medicine, \\ Jacksonville, Florida
}

OBJECTIVE The transition of the young adult with spina bifida (YASB) from pediatric to adult health care is considered a priority by organized pediatrics. There is a paucity of transition programs and related studies. Jacksonville Health and Transition Services (JaxHATS) is one such transition program in Jacksonville, Florida. This study's purpose was to evaluate the health care access, utilization, and quality of life (QOL) of a group of YASBs who have transitioned from pediatric care.

METHODS A survey tool addressing access to health care and quality of health and life was developed based on an established survey. Records of the Spinal Defects Clinic held at Wolfson Children's Hospital and JaxHATS Clinic were reviewed and YASBs (> 18 and $<30$ years old) were identified.

RESULTS Ten of the 12 invited YASBs in the Jacksonville area completed the surveys. The mean age of respondents was 25.1 years. All reported regular medical home visits, 8 with JaxHATS and 2 with other family care groups. All reported easy access to medical care and routine visits to spina bifida (SB) specialists; none reported difficulty or delays in obtaining health care. Only 2 patients required emergent care in the last year for an SB-related medical problem. Seven respondents reported very good to excellent QOL. Family, lifestyle, and environmental factors were also examined.

CONCLUSIONS In this small group of YASBs with a medical home, easy access to care for medical conditions was the norm, with few individuals having recent emergency visits and almost all reporting at least a good overall QOL. Larger studies of YASBs are needed to evaluate the positive effects of medical homes on health and QOL in this population.

http://thejns.org/doi/abs/10.3171/2015.7.PEDS14694

KEY WORDS spina bifida; pediatric neurosurgery; transition services; pediatrics; young adults; congenital

A DVANCES in clinical care have enabled a greater number of individuals with spina bifida (SB) to live well into adulthood. ${ }^{14,15} \mathrm{~A}$ vital part of the continuum of care for those with SB is the transition from pediatric to adult health care systems, the importance of which has been recognized by both organized pediatric neurosurgery and pediatrics. ${ }^{10}$ Associated with this transition, however, are a number of obstacles, many of which entail the need for both coordinated and consistent access to primary and specialty health care. ${ }^{12}$ There are several transition programs in the country aimed at providing such care to these individuals. In Jacksonville, Florida, the Spinal Defects Clinic (SDC) at Wolfson Children's Hospital (WCH) provides pediatric care for children with SB. As they approach the age of 18 , patients are transitioned out of the pediatric program to adult care services. The Jacksonville Health and Transition Services (JaxHATS) Program of the University of Florida (UF) Health Jacksonville is one such program that deals with this health care transition of patients from the WCH SDC and provides a medical home for young adults with chronic diseases of childhood. Few studies have described the process of transition in their programs. ${ }^{9,12}$ Moreover, few studies have assessed, empirically, the impact of this transition to adult-centered health care in terms of health care outcomes. ${ }^{2}$ The purpose of this pilot study is to evaluate the health care access and utilization in the Jacksonville, Florida, area of young adults with spina bifida (YASBs) after transfer to adult

ABBREVIATIONS JaxHATS = Jacksonville Health and Transition Services; $Q \mathrm{QL}=$ quality of life; $\mathrm{SB}=$ spina bifida; SDC = Spinal Defects Clinic; UF = University of Florida; $W C H=$ Wolfson Children's Hospital; YASB = young adult with spina bifida.

SUBMITTED January 5, 2015. ACCEPTED July 13, 2015.

INCLUDE WHEN CITING Published online October 23, 2015; DOI: 10.3171/2015.7.PEDS14694. 
care, as well as their quality of life (QOL) after transfer to adult care.

\section{Methods \\ Patient Selection}

Following approval by the UF Jacksonville Institutional Review Board, previous SB patients at the SDC who were between 20 and 26 years of age were sought out, regardless of their transition status. Medical records of the SDC and the JaxHATS clinic were used in this search. Invitations were sent via mail to those with valid addresses in the 5 counties (Baker, Clay, Duval, St. Johns, Nassau) served by WCH.

\section{Survey Tool}

A survey tool was developed to evaluate demographic characteristics, educational and employment history, access to adult health care, and QOL. The QOL assessment was adapted from the SF-36 (36-Item Short Form Health Survey), the most widely used and validated QOL measurement tool. Additional questions regarding the study participants' utilization of health care resources were included in the survey. A member of the research team assisted respondents (via telephone) in the answering of the survey questions. A copy of the 24-page survey can be requested from the corresponding author.

\section{Transition Process}

In North Florida and Southeast Georgia, children with $\mathrm{SB}$ receive care from the multidisciplinary SDC at WCH. This monthly clinic is staffed by faculty pediatric neurosurgeons, physiatrists, and a developmental pediatrician from the UF College of Medicine Jacksonville, and urologists, orthopedic surgeons, and gastroenterology specialists from the Nemours Children's Clinic Jacksonville, as well as physical therapists and a social worker from $\mathrm{WCH} .{ }^{1}$ Additional social services support is provided by Children's Medical Services. The family and patient are made aware of the transition process to JaxHATS as the child enters high school. The formal transition process starts between the ages of 16 and 18 years, and patients need to be medically and socially stable during the transition. Patients are typically evaluated in the SDC for a period of at least 2 years prior to their transfer to the JaxHATS clinic. There is a 1-2-year bridging period, where the patients are seen at both the WCH SDC and JaxHATS clinic, until they are transferred out of the WCH SDC to JaxHATS. The JaxHATS clinic transfers the patients completely to adult care services when the enrolled patients are consistently seeing the appropriate adult specialists and are taking independent responsibility for their health and health care needs.

\section{JaxHATS as a Medical Home}

Established in 2005, the JaxHATS program provides a primary care medical home to young adults during healthcare transition and is staffed by board-certified internal medicine and pediatric physicians from UF College of Medicine Jacksonville, a nurse care coordinator, and a transition coordinator. JaxHATS provides transition- oriented primary care and other support to patients with a wide range of conditions that arise in childhood, including SB, sickle cell anemia, cystic fibrosis, congenital heart disease, and other childhood diseases. As a transition medical home, JaxHATS provide continuity of primary care, referral and coordination of specialty care, longitudinal disease management, acute care, and special "transition" visits. During transition visits, the teams assess the transition readiness of all youth in the program; provide ongoing training for disease self-management and how to access and use health care services; provide education and support to address other transition-related needs such as insurance issues, guardianship, referral to worktraining programs, advocacy in the schools for transition individualized education plan support, etc. The primary care providers of JaxHATS coordinate the specialty care of the YASBs within the UF Health Jacksonville system, which, as a system, accepts all forms of insurance, including Medicaid.

\section{JaxHATS Providers}

One of the primary care providers in JaxHATS (D.W.) was the first primary care pediatrician for the WCH SDC, and in that role became familiar with the special needs of youth and YASBs, such as their neurocognitive challenges, signs of shunt malfunction, bowel and bladder management, and skin care. This pediatrician helped to train the internal medicine providers and residents in the primary care of patients with SB. At the start of the JaxHATS program, the Spina Bifida Association hosted a meeting between pediatric and adult specialists who care for children with SB and YASBs (primary care [JaxHATS], neurosurgery, gastroenterology, urology, and orthopedics). The meeting resulted in agreement from the adult specialists to accept referrals of patients from the pediatric specialists. The adult specialists were mainly faculty members of the UF College of Medicine Jacksonville. A key element of the referral or transfer process was that JaxHATS would continue to follow the patients and monitor their disease self-management, facilitate adherence with appointments, help with other referrals, and assist with lapses in insurance or other issues that might arise.

\section{Results}

\section{Patient Characteristics}

Between 2010 and 2014, the average number of patients followed at the SDC annually was 87 .

After searching the SDC records and the JaxHATS database, we identified 27 former patients (regardless of their transition status), between the ages of 20 and 26 years, who had addresses in the Jacksonville metropolitan area. This includes Duval County (the boundaries of the city of Jacksonville) and its 4 adjacent counties. Twelve patients with valid addresses were contacted by mail and invited to participate in this study; 10 patients ( 3 male, 7 female) consented and completed surveys. The mean age of the respondents was 25.1 years.

\section{Disease Characteristics}

All but 1 patient had myelomeningocele and shunted hydrocephalus; 1 patient did not have hydrocephalus. Pa- 
tients were asked to rate specific SB-related conditions according to severity of disease. Skin-related conditions (pressure sores and latex allergy) were the most severe in the largest number of respondents, followed by bladder control, mobility, and balance (Table 1).

\section{Socioeconomics}

All respondents indicated they did not have a spouse or partner. Nine identified their parents as being supportive of their condition. Seven patients had completed high school, and 2 had completed college. Six patients were attending some form of educational program at the time of the survey, and another was participating in an adult training program. Two patients reported being involved in some form of vocational activity, and 1 patients was limited to bed rest due to a wound infection.

\section{Income}

Only 1 patient received income from employment, and the remaining patients received social security and/or disability benefits. Monthly income was extremely low, with 2 respondents reporting a monthly income of less than $\$ 400$, and 5 reporting a monthly income between $\$ 400$ and $\$ 1000$. The highest monthly income, between $\$ 1100$ and $\$ 2500$, was reported by the only employed respondent.

\section{Insurance Status}

Five patients had insurance coverage through Medicaid, Medicare, or both. Three patients had military insurance (TRICARE), or Medicaid, or both; 1 patient had private insurance; and 1 patient was uninsured.

\section{Access to Medical Care and Medical Home Utilization}

All patients reported having a medical home, where they had regular access to either a doctor's office or health center, in which they received primary care when they were well or ill. Six patients received primary care services through JaxHATS, 3 patients received their care at private practice clinics, and 1 patient received care at the Mayo Clinic Jacksonville. None of the patients reported any difficulty, delay, or denial in receiving health care in the past 12 months.

All patients reported being able to routinely see specialists for SB-related problems through their medical homes. These problems included those listed in Table 1. Eight patients received this specialty care through JaxHATS, 1 patient received care at a private practice clinic, and the other received care at the Mayo Clinic Jacksonville.

\section{Utilization of Hospital Care}

For SB-related medical complications within the last 12 months, only 2 patients reported they needed to go to hospitals for emergent care. More than double the number of respondents (5) required utilization of emergent care for non-SB-related medical complications. Five patients required inpatient hospitalization for SB-related problems, and 4 required inpatient hospitalization for non-SB-related problems.

\section{Quality of Life}

Subjective ratings in combination with a free-response
TABLE 1. Burden of disease in 10 adult patients with SB*

\begin{tabular}{lcc}
\hline \multicolumn{1}{c}{ Condition } & None/Mild & Moderate/Severe \\
\hline Latex allergy & 3 & 7 \\
\hline Pressure sores & 4 & 6 \\
\hline Bladder control & 5 & 5 \\
\hline Mobility & 5 & 5 \\
\hline Balance & 5 & 5 \\
\hline Pain & 7 & 3 \\
\hline Skin breakdown & 7 & 3 \\
\hline Scoliosis & 7 & 3 \\
\hline Weight & 8 & 2 \\
\hline Endurance & 9 & 1 \\
\hline Strength & 10 & 0 \\
\hline Osteoporosis & 10 & 0 \\
\hline Breathing & 10 & 0 \\
\hline Injuries & 9 & 0 \\
\hline Other & 8 & 2 \\
\hline
\end{tabular}

* Patients were asked to rate severity of the following SB-related conditions as none, mild, moderate, severe, don't know, or refused. The none/mild/don't know responses were pooled, as were the moderate or severe responses.

section were used to assess the respondents' QOL. Five patients reported QOL to be "excellent," 2 reported "very good," 2 gave a rating of "good," and 1 reported "fair." Selected free responses considered to be representative of positive subjective ratings for QOL included "I don't have to go to the doctor/hospital as much as I used to," "I have the best family," "my mother," "living alone," "driving to work," "great friends," "having a sense of humor," and "having a good education."

Transportation availability and occupation data were reported in the survey as well. One patient found the availability of transportation for medical use to be a problem, 2 patients encountered difficulty with its occupational use, and 6 respondents reported difficulty with its general use. Although 8 patients reported their condition does not keep them from working, housekeeping, or going to school, 6 respondents did report that these same activities are limited by SB. At least 8 respondents reported expectations of going to college, driving an automobile, and/or being employed.

\section{Discussion}

The primary purpose of this study was to evaluate the health care access and utilization in YASBs. In this small survey, where all respondents belonged to medical homes, we found that all reported good access to primary and specialty care, with no delays or denials in medical care.

The transition of SB patients from pediatric to adult health care systems has been considered a priority by organized pediatric neurosurgery and pediatrics. ${ }^{10}$ Reports from transition programs in other countries have detailed their preliminary results. ${ }^{12}$

Regarding the profile of the patients surveyed, they were young (mean age 25.1 years), unmarried, and living in the Jacksonville area. Regardless of their abovementioned insurance, one of the advantages of having the 
JaxHATS program based within the UF Health Jacksonville system is that it provides care to both insured and uninsured patients, which facilitates access to care for YASBs regardless of their ability to pay. Moreover, the JaxHATS program, acting as the medical home, connected the patients initially to needed specialty care (urology, neurosurgery, and gastroenterology) and helped them to maintain these connections. Moreover, there is a defined and planned transition and overlap between the multidisciplinary pediatric SDC and JaxHATS, which is likely one of the reasons why 8 of the 10 patients surveyed received their specialty care through UF Health Jacksonville.

The care of a patient with SB requires support from a number of different medical specialties. ${ }^{5}$ While utilizing a medical home, our respondents reported access to primary and specialized care that was both consistent and timely, without any delay. In contrast, a study of 122 individuals with SB between the ages of 16 and 59 found that $71 \%$ of them reported difficulty accessing health care, which was associated with worse overall health. ${ }^{5}$ Prompt access to medical care is a relevant concern, particularly in $\mathrm{SB}$, where the number of adult primary care and specialty physicians with extensive knowledge of this disease has been shown to be insufficient. ${ }^{8}$ Such inexperience has been identified as a clear barrier to a successful transition for patients with SB. ${ }^{2}$ One qualitative study involving SB patients noted that some of their physicians were uncomfortable or ambivalent about certain topics, such as communicating bad news, and some physicians felt they lacked specific training. Uninterrupted access to health care was found to be a critical component in maintaining the health and well-being of the SB patient population as they transition from pediatric to adult health care. ${ }^{6}$ Transition to the JaxHATS program ensured both continuous and uninterrupted access to both primary care and specialty providers with expertise in the care of YASB.

Literature on the utilization of hospital care for patients with SB who belong to a transitional medical home has been scant. ${ }^{2}$ It has been established that individuals with SB use health care services to a greater extent than the normal population. ${ }^{4,16}$ After examining the utilization of acute medical and inpatient services over a 12-month period in our group of 10 respondents, we found that twice as many of them used emergency care services for non-SB-related conditions than for SB-related conditions. This may be a reflection of our respondents' reported easy access to specialty care, either through JaxHATS and UF Health Jacksonville (8 of 10) or the Mayo Clinic Jacksonville (1 of 10). Furthermore, almost an equal number of patients reported being hospitalized for SB- or non-SB-related conditions, 5 and 4 patients, respectively, although it is unclear if these hospital admissions were elective or emergent. Young et al. examined the health care utilization of young adults with chronic neurological disabilities (including SB) and found that these individuals used health care, both inpatient and outpatient, more than 9 times more than the normal population. ${ }^{17}$ This study also found that the number of emergency visits were fewer for patients who had a primary care provider. It is known that specialized medical transition homes provide for the coordination and efficient provision of primary care (along with other services like nursing and social work) necessary for this medically complex condition. Patients and their families are spared repeated visits, and the quality of their care is enhanced by continuity and communication among these healthcare professionals. ${ }^{12}$ In addition to the coordination of primary care, medical homes assure access to specialty care by making and following up on referrals to adult specialist. This may explain the low utilization of acute care services for SB-related conditions among our respondents. When examining the effects of a medical home on the health of SB patients, a study by Mukherjee showed an increase in the number of preventable surgeries for a group of YASBs who no longer had their medical care coordinated by a specialized medical home that had disbanded. ${ }^{8}$

The favorable access and low acute medical care utilization reported by our study group support the hypothesis that belonging to a medical home enhances the quality of care of YASBs. Medical homes, with their concentration of various medical services, both primary and specialized, provide an enhanced level of medical care access to our YASBs. ${ }^{12}$ However, the nature of the relationship between the use of a medical transition home by our group and their reported quality of medical care is not yet fully understood and is the subject of future investigation. Nonetheless, the improved coordination and timely provision of medical care in our study group is believed to play a role in our group's quality of care.

The majority of our study participants (7 of 10) reported a favorable QOL (either "very good" or "excellent"). A contributing factor to these favorable ratings may be the ease of access to primary and specialty care provided by the patients' medical home. Similarly, in a study on the satisfaction of a group of YASBs with their transition into adulthood, Boudos and Mukherjee found shorter appointment times and increased independence with health care management to be favorable outcomes in the process (Boudos RM, Mukherjee S: Satisfaction with transition to adult health care: a focus group experience with adults and parents. Presentation to the Second World Congress on Spina Bifida Research and Care, Las Vegas, NV, March 11-14, 2012). Other studies have shown that medical problems that occur as a complication due to SB impose limitations on physical and social-related activities, which in turn negatively impact the QOL..$^{13}$ The medical homes in this study, by possibly reducing or preventing problems due to SB-related conditions, may have positively affected the patients' QOL; however, further study is needed to confirm this.

There are other components of QOL important to SB patients. Castree and Walker found their study group of YASBs to be considerably handicapped in 3 important domains thought to be related to QOL: independence, occupation, and social contact with peers. ${ }^{3}$ These domains are similarly reflected in the goals reported by our respondents, where a majority ( 8 of 10$)$ reported goals related to a need for independence and/or occupation, such as going to college, driving an automobile, or being employed.

Transportation, which can affect all 3 of the abovementioned QOL domains, was found in our group to not be a problem when needed for medical or occupational use. This may partly be due to the availability of county funding support for medical transportation. However, the majority of our respondents (6 of 10) reported that trans- 
portation, as it pertained to personal use, was an issue. Transportation for personal use may involve traveling to attend social activities or other personal activities rather than to attend medical appointments or occupations. This sentiment was voiced by a survey respondent, who expressed the need for more social opportunities in the Jacksonville area. The need for more social interaction was, in fact, one of the desires expressed in a similar study on the transition of YASBs. ${ }^{11}$ As young adults, YASBs are encouraged to actively participate in their decision-making so as to direct their own healthcare, leading to an effective transition of care. In addition to social interaction, independence has been identified as a necessary factor for successful care transition. ${ }^{2}$ These 2 factors are affected by access to transportation.

The extent to which medical home utilization has improved the QOL in our patients is difficult to estimate given this study's limitations. Although there are many factors that impact a patient's assessment of their respective QOL, we believe the QOL of our group of YASBs has been positively influenced by the improvement of their quality of care via the use of medical homes. The relationship between medical home utilization and other factors of QOL should be a subject of future investigation.

The limitations of our study pertain to its small sample size and the lack of control participants, as only 10 patients were able to participate. Recruitment of patients was challenging, particularly in obtaining accurate addresses for them. Despite our efforts to be inclusive of SDC patients regardless of their transition status, only patients who were engaged in the transition process responded to our recruitment. We realize that this selection bias limits the impact of the findings in this paper. Furthermore, the self-reporting nature of the survey may limit the quality of responses as these are almost completely dependent on respondent interpretation of the questions and their knowledge of the answers. These limitations make it difficult to generalize our findings or to effectively compare them with those of other studies. Nevertheless, the findings in this pilot study can serve as a guide in the design of larger multicenter studies.

\section{Conclusions}

In this survey of a small sample of young adults with $\mathrm{SB}$, a medical home provided access to primary and specialty care for all respondents. Having a comprehensive medical home may have contributed to the group's low utilization of emergency care for SB-related problems as well as the majority of the group reporting a favorable QOL, despite their lower socioeconomic status. Studies involving larger groups are needed to determine further the relationship between accessing a medical home with the outcomes of SB in adulthood.

\section{References}

1. Aldana PR, Wood DL, Postlethwait RA, James HE: Initiating, developing and evaluating a comprehensive spinal defects clinic: a clinical report. Pediatr Neurosurg 46:329_ 334, 2010

2. Binks JA, Barden WS, Burke TA, Young NL: What do we really know about the transition to adult-centered health care?
A focus on cerebral palsy and spina bifida. Arch Phys Med Rehabil 88:1064-1073, 2007

3. Castree BJ, Walker JH: The young adult with spina bifida. Br Med J (Clin Res Ed) 283:1040-1042, 1981

4. Dicianno BE, Wilson R: Hospitalizations of adults with spina bifida and congenital spinal cord anomalies. Arch Phys Med Rehabil 91:529-535, 2010

5. Liptak GS, El Samra A: Optimizing health care for children with spina bifida. Dev Disabil Res Rev 16:66-75, 2010

6. Lotstein DS, McPherson M, Strickland B, Newacheck PW: Transition planning for youth with special health care needs: results from the National Survey of Children with Special Health Care Needs. Pediatrics 115:1562-1568, 2005

7. McDonagh JE: Growing up and moving on: transition from pediatric to adult care. Pediatr Transplant 9:364-372, 2005

8. Mukherjee S: Transition to adulthood in spina bifida: changing roles and expectations. ScientificWorldJournal 7:18901895,2007

9. Peterson PM, Rauen KK, Brown J, Cole J: Spina bifida: the transition into adulthood begins in infancy. Rehabil Nurs 19:229-238, 1994

10. Piatt JH Jr: The Section on Neurological Surgery of the American Academy of Pediatrics: Annual Report 2005 to 2006. J Neurosurg 106 (4 Suppl):324-327, 2007

11. Ridosh M, Braun P, Roux G, Bellin M, Sawin K: Transition in young adults with spina bifida: a qualitative study. Child Care Health Dev 37:866-874, 2011

12. Sawyer SM, Collins N, Bryan D, Brown D, Hope MA, Bowes G: Young people with spina bifida: transfer from paediatric to adult health care. J Paediatr Child Health 34:414-417, 1998

13. Simeonsson RJ, McMillen JS, Huntington GS: Secondary conditions in children with disabilities: spina bifida as a case example. Ment Retard Dev Disabil Res Rev 8:198-205, 2002

14. Stewart DA, Law MC, Rosenbaum P, Willms DG: A qualitative study of the transition to adulthood for youth with physical disabilities. Phys Occup Ther Pediatr 21:3-21, 2001

15. Viner R: Barriers and good practice in transition from paediatric to adult care. J R Soc Med 94 (Suppl 40):2-4, 2001

16. Wilson R, Lewis SA, Dicianno BE: Targeted preventive care may be needed for adults with congenital spine anomalies. PM R 3:730-738, 2011

17. Young NL, Steele C, Fehlings D, Jutai J, Olmsted N, Williams JI: Use of health care among adults with chronic and complex physical disabilities of childhood. Disabil Rehabil 27:1455-1460, 2005

\section{Disclosure}

The authors report no conflict of interest concerning the materials or methods used in this study or the findings specified in this paper.

\section{Author Contributions}

Conception and design: Aldana, Aguilera, James. Acquisition of data: Aguilera, Keeley. Analysis and interpretation of data: Aguilera, Wood. Drafting the article: Aguilera. Critically revising the article: Aldana, Aguilera, Wood. Reviewed submitted version of manuscript: all authors. Approved the final version of the manuscript on behalf of all authors: Aldana. Administrative/technical/ material support: Aldana, Keeley, James. Study supervision: Aldana.

\section{Correspondence}

Philipp R. Aldana, Division of Pediatric Neurosurgery, UF College of Medicine Jacksonville, 836 Prudential Dr., Ste. 1205, Pavilion Bldg., Jacksonville, FL 32207. email: philipp.aldana@ jax.ufl.edu. 